\title{
Percepção da ciência entre professores do ensino médio: comparação do consumo de informação científica entre docentes, estudantes e população de São Paulo
}

\author{
Márcia Azevedo Coelho, Universidade Estadual de Campinas, Brasil \\ Ana Paula Morales, Universidade Estadual de Campinas, Brasil \\ Carlos Vogt, Universidade Estadual de Campinas, Brasil
}

\begin{abstract}
Resumo: As pesquisas de percepção pública da ciência (PPC) têm se consolidado na Ibero-América nos últimos anos, fortalecendo a linha de pesquisa e desenvolvendo um padrão metodológico regional (VOGT \& POLINO, 2003). Estudos nesse sentido têm sido realizados em diversos países, com diferentes públicos. Entre 2013 e 2016, foi realizada a pesquisa de Percepção de Professores de Ensino Médio do Estado de São Paulo sobre Temas Relacionados a Ciência e Tecnologia $(C \& T)$ que, por meio do Indicador de Consumo de Informação científica (Icic), permitiu relacionar as práticas informacionais de docentes sobre temas de $C \& T$ com questões relativas a atitudes, interesse, valoração e apropriação de temas científicos, bem como com a prática pedagógica em sala de aula. Este artigo objetiva promover a reflexão acerca do consumo de informação científica do professor em relação a outros atores importantes no processo de circulação do conhecimento, como jovens estudantes e cidadãos em geral, a fim de averiguar se a formação docente influencia no nível de consumo de informação sobre $C \& T$ e se diferencia esse profissional de outros atores sociais, no que concerne à percepção de assuntos relacionados a C\&T e sua influência na e para a sociedade.
\end{abstract}

Palavras chave: percepção pública da C\&T, consumo de informação científica, práticas pedagógicas, cultura científica

Abstract: Research on Public Understanding of Science (PUS) has been established in Ibero America in recent years, strengthening the research area and developing a regional methodological standard (VOGT \& POLINO, 2003). Between 2013 and 2016, we conducted the study Perception of School Teachers of the State of São Paulo on issues related to science and technology $(S \& T)$, that through the Scientific Information Consumption Indicator (Icic), allowed to relate teachers' practices on $S \& T$ information with issues related to attitudes, interest, valuation and appropriation of scientific themes, as well as the pedagogical practice in the classroom. This article aims to promote reflection on teachers' scientific information consumption in relation to other key players in the process of circulation of knowledge, such as young students and citizens in general, in order to ascertain whether a teacher training influences the level of information consumption on $S \& T$ and differentiates this professional from other social actors, concerning the perception of matters related to $S \& T$ and its influence on and for society.

Keywords: Public Understanding of Science, Scientific Information Consumption, Pedagogical Practices, Scientific Culture

\section{Introdução}

$\mathrm{E}$ ste artigo apresenta alguns resultados da pesquisa Percepção dos Professores de Ensino Médio do Estado de São Paulo sobre Temas Relacionados a Ciência e Tecnologia (PPEMCT), realizada entre 2013 e 2016, em comparação a resultados obtidos em pesquisas anteriores sobre o mesmo tema realizadas com a população geral (FAPESP, 2011) e com jovens estudantes (OEI, 2011; Gouw, 2013), também no estado de São Paulo, Brasil.

A análise objetiva avaliar, prioritariamente, a correlação entre o nível de cultura científica do professor e sua posição frente a temas relacionados a C\&T, avaliando se o conhecimento adquirido pelos docentes em sua formação e exercício profissional os tornam cidadãos com percepção diferenciada da população em geral acerca desses mesmos autores.

Revista Internacional de Aprendizaje en Ciencia, Matemáticas y Tecnología

Volumen 3, Número 1, 2016, <http://sobrelaeducacion.com>, ISSN 2386-8791

(C) Global Knowledge Academics. M. Azevedo Coelho, A. Paula Morales e C. Vogt.

Todos los derechos reservados. Permisos: soporte@gkacademics.com 


\section{Metodologia}

A pesquisa PPMCT foi desenvolvida por meio de aplicação de survey, de autopreenchimento anônimo, criado no Google Docs, com link enviado para o correio eletrônico (e-mail) de professores do ensino médio das redes pública e privada da cidade e estado de São Paulo.

A amostra analisada neste trabalho é composta por parte do universo da pesquisa, a saber: professores de ensino médio vinculados à Secretaria de Educação do Estado de São Paulo (SEESP), atuantes na rede regular e na Fundação Casa (SEESP-FC), ao Sindicato dos Professores de São Paulo (SINPRO-SP) e ao Instituto Federal de Educação, Ciência e Tecnologia de São Paulo (IFSP), somando 9203 respondentes.

O questionário aplicado aos professores do ensino médio foi estruturado a partir de perguntas de quatro tipos: (i) elaboradas especificamente para a pesquisa; (ii) reutilizadas de indicadores nacionais e internacionais com amplo reconhecimento em pesquisas de percepção pública da ciência (COLCIENCIAS, 2005; SECYT, 2007; MCTI, 2006; FECYT, 2004-2006; RICYT, OEI, FECYT, 2007; CETIC, 2011); (iii) adaptadas dos questionários dos indicadores supracitados; e (iv) reutilizadas do questionário elaborado por Rocha (2013).

As questões foram distribuídas em quatro eixos e seis indicadores, apresentados a seguir:

1. Interesse e informação em C\&T

1.1 Interesse dos jovens na carreira científica

1.2 Hábitos informativos e culturais sobre ciência e tecnologia (C\&T)

2. Valorações e atitudes sobre $C \& T$

2.1 Imagem da ciência e da tecnologia (C\&T)

2.2 Imagem sobre os cientistas e sua profissão

3. Apropriação individual e social de C\&T

3.1 Prática docente e sua relação com ciência, tecnologia e interdisciplinaridade

4. Cidadania e políticas de C\&T

4.1 Conhecimento do sistema institucional da C\&T

Para este artigo, foram selecionadas, de questionários aplicados no estado de São Paulo: três questões respondidas por professores do ensino médio (Coelho, 2014); três questões da pesquisa realizada com a população em geral (FAPESP, 2011) e uma questão de duas pesquisas realizadas com jovens estudantes de ensino médio (OEI, 2011; Gouw, 2013).

Tal seleção permitiu que fossem estabelecidas comparações entre as respostas da população em geral, dos jovens estudantes e dos professores, com a finalidade primordial de avaliar se há diferenças significativas entre a percepção e a compreensão da importância do desenvolvimento científico e tecnológico entre esses diferentes atores do processo de circulação do conhecimento.

Os resultados apresentados são relativos às perguntas que compõem os eixos: "Interesse e informação em C\&T" e "Valorações e atitudes sobre C\&T" e os indicadores: "Hábitos informativos e culturais sobre ciência e tecnologia (C\&T)" e "Imagem da ciência e da tecnologia (C\&T)", respectivamente.

O objetivo das questões pertencentes ao indicador: "Hábitos informativos e culturais sobre ciência e tecnologia (C\&T)" é, prioritariamente, aferir o interesse autodeclarado dos entrevistados sobre temas relacionados em a C\&T e relacioná-lo ao "Indicador de Consumo de Informação Científica" (Icic).

\section{Indicador de Consumo de Informação Científica}

O Indicador de Consumo de Informação Científica (Icic) foi proposto pela Secretaria de Ciencia y Técnica da Universidad de Buenos Aires (Secyt), aplicado na Primera Encuesta Nacional de Percepción Pública de la Ciencia, em 2003, e aperfeiçoado em 2007, na Segunda Encuesta Nacional de Percepción Pública de la Ciencia. Esse indicador determina o índice de consumo de informação científica declarado pelos entrevistados e possibilita análises que avaliam o quanto o consumo de informação pode ou não afetar atitudes e percepção dos sujeitos, estabelecer relações 
com o conhecimento em ciência e tecnologia da população, estar ligado a determinados hábitos de participação em C\&T, ou mesmo relacionar-se de maneira direta com atitudes e imagens que se solidificam em relação ao papel da ciência e do cientista.

Na pesquisa de percepção dos professores aqui apresentada, esse índice foi constituído por sete perguntas de consumo informativo que abordam a frequência com que o docente realiza as seguintes ações, quando não está trabalhando: i) assiste a filmes de ficção científica, ii) assiste a programas ou documentários na televisão sobre natureza e vida animal; vê notícias sobre ciências em iii) jornais e iv) programas televisivos sobre C\&T, v) realiza leitura de livros ou HQs de divulgação científica, vi) visita museus, centros ou exposições sobre $C \& T$; e vii) ouve programas radiofônicos sobre $C \& T$.

A cada resposta foi atribuído o valor máximo de 1 , com variação de 0,2 para cada frequência de consumo, estabelecendo os seguintes valores: Nunca $=0$; Anual $=0,2$; Semestral $=0,4$; Bimestral $=0,6 ;$ Mensal $=0,8 ;$ Semanal $=1$.

O Icic se constitui pela média simples das respostas às sete questões e a análise desses valores se dá a partir de cinco estratos, definidos em Secyt (2007):

Tabela 1: Categorias do Indicador de Consumo de Informação Científica (Icic)

\begin{tabular}{|l|l|}
\hline Valor Icic & Categoria Icic \\
\hline 0 & nulo \\
\hline $\mathrm{x}<0,25 \mathrm{e} \neq 0$ & baixo \\
\hline $0,25 \leq \mathrm{x}>0,5$ & médio-baixo \\
\hline $0,5 \leq \mathrm{x}>0,75$ & médio-alto \\
\hline $0,75 \leq \mathrm{x} \geq 1$ & alto \\
\hline
\end{tabular}

Para este artigo foram comparados o nível de Icic dos docentes: vinculados à Secretaria do Estado de São Paulo (SEESP), ao Sindicato dos Professores de São Paulo (SINPRO); e ao Instituto Federal de São Paulo (IFSP).

Também foi apresentado o nível médio de Icic dos professores em comparação à média obtida na pesquisa com a população em geral de sete capitais ibero-americanas.

\section{Resultados}

Comparando os níveis de Icic dos docentes vinculados às redes estadual, federal e privada de ensino, é possível notar que, embora haja diferença significativa entre o nível de Icic dos professores do IFSP e o dos vinculados às demais instituições, a maioria $(84,6 \%)$ dos entrevistados pertence aos grupos de Icic alto e médio-alto.

Gráfico 1: Proporção do Icic entre os professores

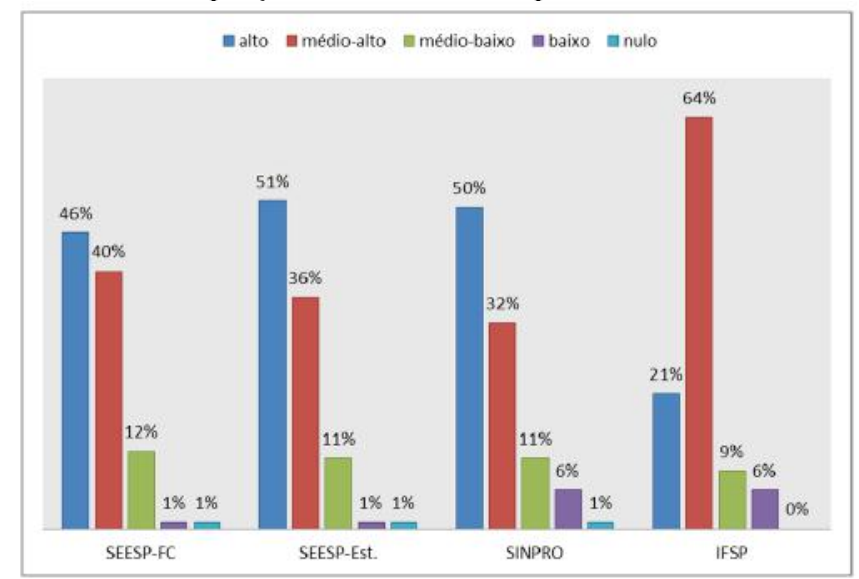

Fonte: Coelho, 2014. 
Do total de professores que responderam ao questionário, 42,3\% apresentam Icic alto, 42,3\% médio-alto, $10,51 \%$ médio-baixo, $3,86 \%$ baixo e $0,57 \%$ nulo. Isso significa que a frequência da realização das práticas de consumo de informação científica apresentadas (assistir a filmes de ficção científica, a programas ou documentários na televisão sobre natureza e vida animal ou C\&T; ler notícias sobre ciências em jornais; realizar leitura de livros ou HQs de divulgação científica; visitar museus, centros ou exposições sobre C\&T; e ouvir programas radiofônicos sobre C\&T) varia de bimestral a semanal para cerca de $85,5 \%$ da amostra.

Gráfico 2: Comparação entre faixas do Icic da população em geral, por cidades (FAPESP, 2011), e dos professores de São Paulo (Coelho, 2014)

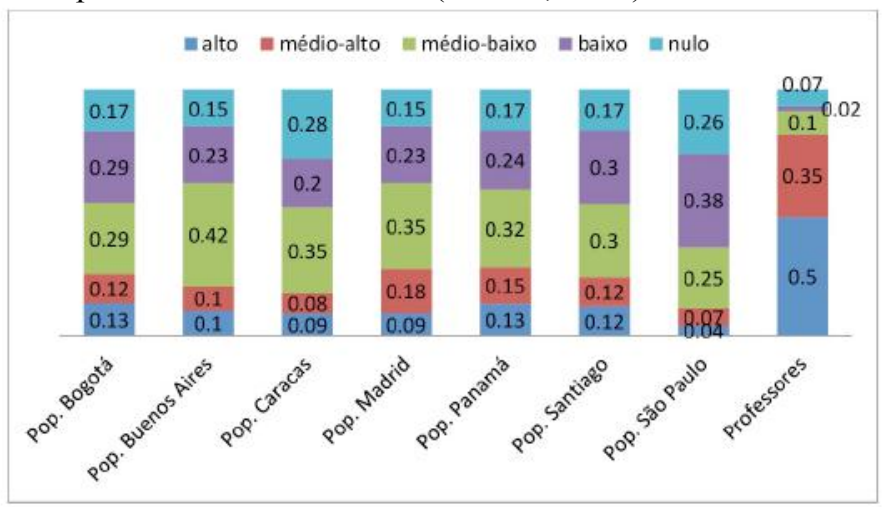

Fontes: Labjor/Unicamp (FAPESP 2011); López Cerezo e Polino (2008); Coelho, 2014.

Comparando o Icic dos professores de ensino médio com o da população em geral de sete capitais ibero-americanas, percebe-se que o grupo de docentes apresenta um nível de consumo de informação científica superior ao da população em geral de todas as capitais em que foi aplicada a pesquisa, como se pode observar no gráfico 2 .

Uma possível explicação para o perfil encontrado na amostra poderia ser que a própria metodologia da pesquisa tenha selecionado professores de Icic alto e médio-alto, pelo fato de o questionário ter sido enviado por e-mail. Ou seja, os professores mais desinformados, com pouco acesso a computadores e os desinteressados podem não ter respondido à pesquisa. Nesse sentido, não podemos afirmar que tais resultados representem a população de professores de ensino médio, na medida em que os respondentes já passaram pelo filtro prévio do interesse.

Considerando a amostra, os dados demonstram que o grupo com Icic alto dos docentes de São Paulo é aprozimadamente 12 vezes maior do que o da população do mesmo estado. Nesse sentido, fica clara a distinção do papel social do professor no processo de circulação do conhecimento, uma vez que caberia a ele, se não produzir - o que seria de fato desejável -, ao menos divulgar o conhecimento pelo ensino e, para tanto, pressupõe-se que o docente possua um nível mais elevado de informação sobre assuntos relacionados ao conhecimento científico do que o cidadão que não teria, necessariamente, a função de divulgar ciência.

Em consequência disso, a fim de observar se a percepção do professor, imbuído de seu papel social no processo de circulação do conhecimento, diferencia-se em algum nível da percepção do cidadão - que muitas vezes se aproxima desses temas apenas por curiosidade/ interesse - foram feitas comparações entre a pesquisa realizada com os docentes de ensino médio e a de Percepção pública da ciência e da tecnologia no estado de São Paulo (FAPESP, 2011), a qual entrevistou o público adulto em geral no mesmo estado brasileiro.

Ainda considerando a importância do professor no processo de circulação do conhecimento e os demais atores que compõem esse sistema, foram cotejados alguns resultados da pesquisa realizada com professores com os da realizada com jovens estudantes de ensino médio da cidade de São Paulo (OEI, 2011), que, por sua vez, são identificados por características próprias, especi- 
ficamente, com as daquele que recebe a informação e "produz" conhecimento por meio do processo de ensino e aprendizagem, estreitamente relacionado à ação do professor.

A questão 24 da pesquisa respondida pelos professores teve o intuito de conhecer a opinião dos docentes acerca do grau de risco e benefício que o desenvolvimento científico pode trazer para a sociedade. Inqueriu-se ao respondente se ele considerava que "a ciência e a tecnologia trazem muitos riscos, alguns riscos, poucos riscos ou nenhum risco".

Os resultados obtidos nessa questão demonstram que a maioria dos docentes de todas as redes de ensino é otimista frente ao desenvolvimento da C\&T. Contudo, as respostas apontam também que o maior número de respondentes $(59,07 \%)$ tem uma confiança reservada nesse desenvolvimento, já que afirma haver mais benefícios do que riscos, por oposição à parcela menor $(13,27 \%)$, absolutamente otimista, que demonstrara confiança excessiva na C\&T, afirmando haver somente benefícios nesse tipo de desenvolvimento.

Essa fração de entusiastas sem restrições, que parece conceber a tecnologia como algo neutro, apartada da sociedade e dos interesses que envolvem todo tipo de investimento no desenvolvimento científico-tecnológico e em seus produtos (BAZZO, 1998, p. 142), entretanto, não constitui a maior parte dos respondentes. A maioria dos professores apresenta atitude ambivalente (GIDDENS, 1991) de reverência e reserva frente à ciência e à aplicação dela.

A maior parcela dos docentes tem um grau de confiança crítico em relação ao desenvolvimento da C\&T, como demonstram os resultados, nos quais: $53,6 \%, 54 \%, 56 \%$ e $72,7 \%$ dos respondentes das redes estadual (Fundação Casa e rede regular), particular e federal, respectivamente, afirmaram que o desenvolvimento da $C \& T$ traz mais benefícios, ainda que não destituído de riscos para a sociedade.

Gráfico 3: Percentual de confiança em C\&T

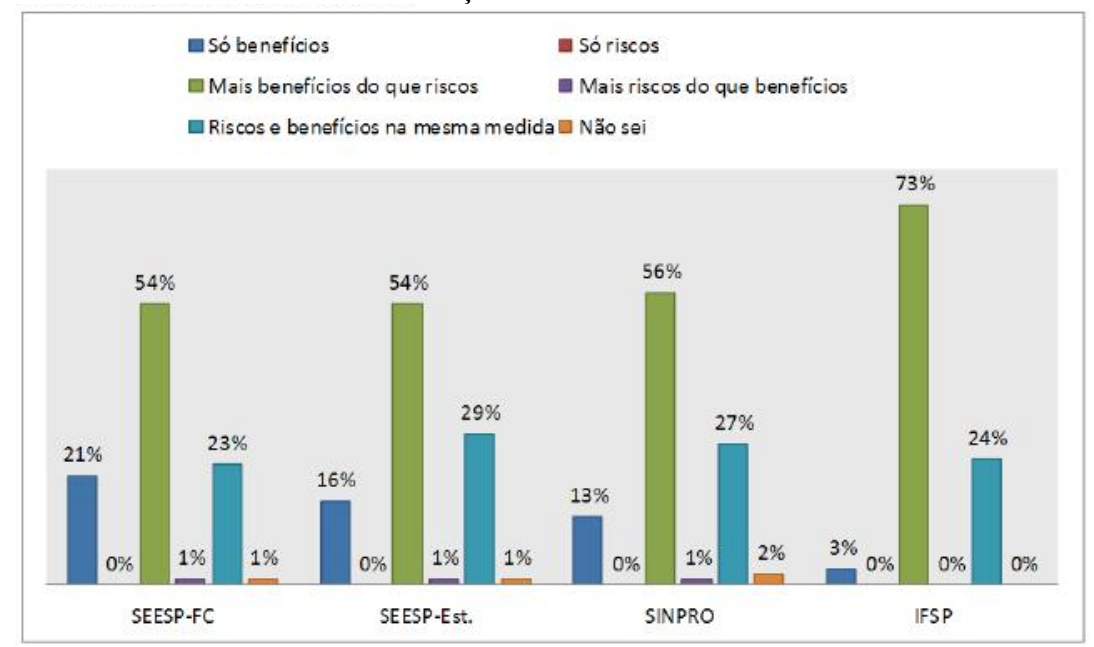

Fonte: Coelho, 2014.

Esse resultado corrobora outros obtidos em pesquisas de percepção pública de âmbito estadual e nacional (FAPESP, 2005, 2011; MCTI, 2010) e também com outras realizadas com jovens estudantes de ensino médio (Gouw, 2013), nas quais grande parte dos respondentes tem a mesma percepção sobre os benefícios da $\mathrm{C} \& \mathrm{~T}$ em relação à sociedade, como demonstra a tabela que segue. 
Tabela 2: Comparação da questão sobre riscos e benefícios entre Fapesp, MCTI e GOUW

\begin{tabular}{|l|c|l|c|l|c|}
\hline \multicolumn{1}{|c|}{ FAPESP (2005) } & $\mathbf{\%}$ & \multicolumn{1}{|c|}{ MCTI (2010) } & \% & \multicolumn{1}{|c|}{ GOUW (2013) } & \% \\
\hline $\begin{array}{l}\text { Os benefícios da } \\
\text { ciência são maiores do } \\
\text { que os efeitos } \\
\text { negativos }\end{array}$ & $72,0 \%$ & $\begin{array}{l}\text { Ciência e tecnologia: } \\
\text { mais benefícios que } \\
\text { malefícios }\end{array}$ & $42,3 \%$ & $\begin{array}{l}\text { Os benefícios da } \\
\text { ciência são maiores do } \\
\text { que os efeitos } \\
\text { negativos que possa ter }\end{array}$ & $52,3 \%$ \\
\hline
\end{tabular}

Fonte: Reproduzido de Gouw (2013).

O enunciado das questões sobre a percepção de riscos e benefícios no questionário aplicado à população geral em 2010 (FAPESP, 2011) apresentava uma pequena diferença: nas perguntas 13 e 14, faziam-se os seguintes questionamentos aos entrevistados, respectivamente: p.13: "Em geral, você acha que nos próximos 20 anos o desenvolvimento da ciência e da tecnologia vai trazer muitos riscos, alguns riscos, poucos riscos ou nenhum risco para o mundo?" e P.14: "Em geral, você acha que nos próximos 20 anos o desenvolvimento da ciência e da tecnologia vai trazer muitos benefícios, alguns benefícios, poucos benefícios ou nenhum benefício para o mundo?”.

Não obstante as diferenças no enunciado, o resultado também apresenta maior percentual entre os que consideram que o desenvolvimento da C\&T trará mais benefícios do que riscos, já que $27,9 \%$ dos respondentes afirmaram perceber muitos riscos e $32,9 \%$, muitos benefícios, conforme demonstrado na tabela 3 .

Tabela 3: Perguntas sobre riscos e benefícios da C\&T em Fapesp, 2011

13. Pergunta: Em geral, você acha que nos próximos 20 anos o desenvolvimento da ciência e da tecnologia vai trazer muitos riscos, alguns riscos, poucos riscos ou nenhum risco para o mundo?

\begin{tabular}{|c|c|}
\hline Resposta & \% \\
\hline Muitos riscos & 27,9 \\
\hline Alguns riscos & 35,7 \\
\hline Poucos riscos & 20,9 \\
\hline Nenhum risco & 9,9 \\
\hline NS/NR & 5,6 \\
\hline
\end{tabular}

14. Pergunta: Em geral, você acha que nos próximos 20 anos o desenvolvimento da ciência e da tecnologia vai trazer muitos benefícios, alguns benefícios, poucos benefícios ou nenhum benefício para o mundo?

\begin{tabular}{|l|c|}
\hline Resposta & \% \\
\hline Muitos benefícios & 32,9 \\
\hline Alguns benefícios & 39,5 \\
\hline Poucos benefícios & 17,6 \\
\hline Nenhum benefício & 6,2 \\
\hline NS/NR & 3,8 \\
\hline
\end{tabular}

Fonte: Fapesp, 2011.

Pelos dados das quatro pesquisas (com jovens estudantes, população em geral e professores) pode-se notar que há, de forma geral, uma perspectiva positiva do papel da ciência e da tecnologia na sociedade.

O fato da concepção edificante da C\&T ter superado a indicação da existência de risco entre os professores, alunos e população em geral corrobora resultados obtidos em outros países. Nos Estados Unidos, segundo relatório da National Science Foundation (2014), a maioria dos americanos (aproximadamente 70\%) disseram que os benefícios da ciência superam os potenciais malefícios e que o governo deveria financiar ainda mais as pesquisas (NSF, 2014).

Entre os chineses, em 2010, 75\% dos entrevistados concordaram "totalmente" ou "parcialmente" que a C \& T trazem mais benefícios do que danos (CRISP, 2010), percentual um pouco mais elevado do que o do Japão, onde $54 \%$ dos entrevistados afirmaram que há mais vantagens do que desvantagens no desenvolvimento da C\&T para a sociedade (NISTEP, 2013). 
Considerando o percentual de pessoas que concordam que a C\&T traz mais benefícios do que riscos para a população, os resultados demonstram que o público em geral de diferentes países, assim como os docentes de São Paulo veem com otimismo o desenvolvimento da ciência e da tecnologia.

Quando questionados sobre o grau de concordância ou discordância de que os cidadãos devem ser ouvidos e suas opiniões consideradas mesmo que não estejam bem informados sobre as implicações da ciência (riscos e benefícios) ou novos desenvolvimentos tecnológicos, a grande maioria dos professores concordou com a participação ativa do cidadão, não obstante o desconhecimento do tema tratado.

Nessas questões (gráficos 4 e 5), as respostas "concordo plenamente" e "concordo parcialmente" foram agrupadas em concordo e as "discordo parcialmente" e "discordo plenamente" em discordo.

A grande maioria dos professores concorda que o cidadão deve ser ouvido e suas opiniões consideradas, ainda que não tenha conhecimento do assunto.

Gráfico 4: Opinião de docentes sobre a importância da participação cidadã em C\&T

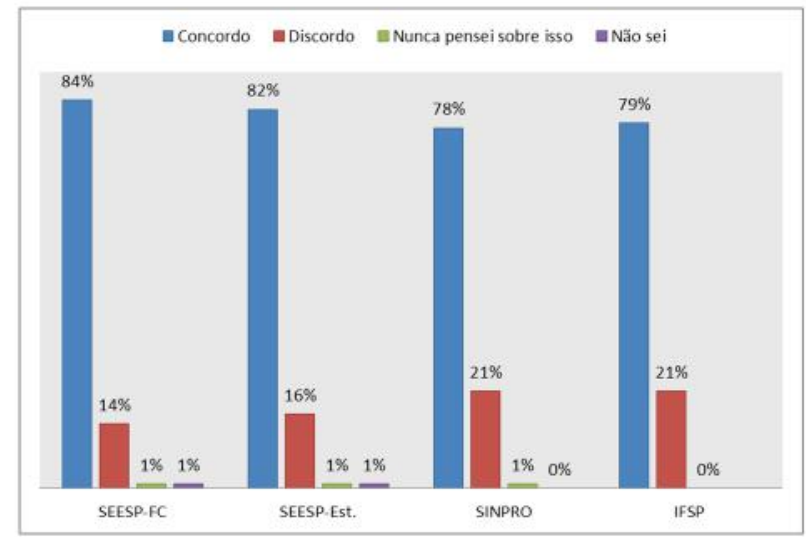

Fonte: Coelho, 2014.

Com poucos pontos percentuais de diferença $(6 \%)$ entre os que mais concordam com a afirmação e os que menos concordam, os docentes da Fundação Casa são os que mais defendem a participação dos cidadãos, seguidos dos professores vinculados à SEESP atuantes na rede regular, ao passo que os docentes sindicalizados do Sinpro apresentem menor índice de concordância, seguidos dos professores do Instituto Federal de Educação, Ciência e Tecnologia.

Em enquete realizada com a população em geral do estado de São Paulo (Fapesp, 2011), a pergunta 22.1 apresentava um enunciado similar - e a partir do qual se elaborou a questão 27.1 da pesquisa com os professores. Nela, o entrevistado deveria dizer se concordava muito, concordava, nem concordava nem discordava, discordava, ou discordava muito com a posição de que "Os cidadãos devem ser ouvidos e sua opinião considerada [em casos de decisões sobre temas relacionados a C\&T]."

Semelhantemente ao resultado obtido na pesquisa com professores, a grande maioria dos entrevistados do estado de São Paulo (89,5\%) afirmou concordar que a participação do cidadão deve ser considerada, independentemente do conhecimento que possa ter sobre o que se propõe a opinar (FAPESP, 2011).

Chama a atenção o percentual de respostas que relevam a necessidade de se conhecer sobre determinado assunto para opinar sobre ele, quando se trata de o respondente ser um professor. Isso pelo fato de o professor pensar, teoricamente, mais sobre a importância do conhecimento para a vida das pessoas e, consequentemente, para a diminuição potencial de decisões erradas. Contudo, pelo resultado, quando se trata da participação social, o conhecimento parece ter menos importância do que a garantia da ação democrática. 
Há ainda que se fazer uma observação sobre essa pergunta 27.1, pois na enquete feita à população em geral, não se apresentava a ressalva de que a população devesse participar, mesmo não estando bem informada sobre o assunto, o que leva a crer que o índice de "concordo" pudesse ter um decréscimo, caso a pergunta tivesse a ressalva, aproximando-se ainda mais do percentual médio de concordância dos entrevistados docentes, que é de $80,65 \%$.

Parece curioso que os profissionais que lidam diretamente com a construção do conhecimento e com a informação considerem-nas pouco relevantes para a participação cidadã, como indicaram os resultados.

Outra possibilidade de interpretação é a de que os respondentes em sua maioria consideram que o direito democrático deva prevalecer sob qualquer outro tipo de condição.

Nesse aspecto, parece não haver diferença entre a percepção daqueles profissionais que lidam diretamente com a informação e a população em geral que, teoricamente, não refletiria, diferentemente do docente, sobre a importância do conhecimento para a prática cidadã.

Constituindo ainda o eixo "Valoração e atitudes sobre C\&T", a pergunta 27 questionava se o entrevistado concordava ou discordava que "É melhor deixar as decisões sobre problemas sociais relacionados à ciência e à tecnologia nas mãos dos especialistas".

Coerentemente com as respostas dadas na questão 27.1, a maioria dos professores afirmou discordar que caberia somente aos especialistas decidir sobre problemas sociais relacionados a C\&T. Contudo, comparando os níveis concordância da questão 27.1 e de discordância da questão 27, percebe-se que alguns resultados demonstram variação inversa. Ou seja, entre os entrevistados, os que mais concordam com a participação cidadã são os docentes da Fundação Casa, seguidos dos professores que atuam na rede estadual regular. Contudo, são esses também os que mais concordam que as decisões devam ser tomadas por especialistas.

De forma mais coerente apresentam-se as posições dos docentes do IFSP, já que o percentual de docentes que afirmaram concordar com a proposição de que o cidadão deve ser ouvido e suas opiniões consideradas, ainda que não tenha domínio sobre o assunto, foi o mesmo $(78,80 \%)$ dos que afirmaram discordar que as decisões de problemas sociais ligados à C\&T deveriam caber somente a especialistas.

Fazendo novamente um comparativo dos resultados obtidos na pesquisa com professores com os da população em geral do estado de São Paulo, pode-se notar que a percepção dos dois grupos mantém certa semelhança, já que entre os entrevistados do segundo grupo (população em geral), 56,4\% afirmaram discordar de que as decisões fiquem somente sob a responsabilidade de especialistas, similar aos resultados do primeiro grupo, dos professores, em que a maioria $(65,65 \%)$ deu a mesma resposta.

\section{Gráfico 5: Percentual de exclusão cidadã em C\&T}

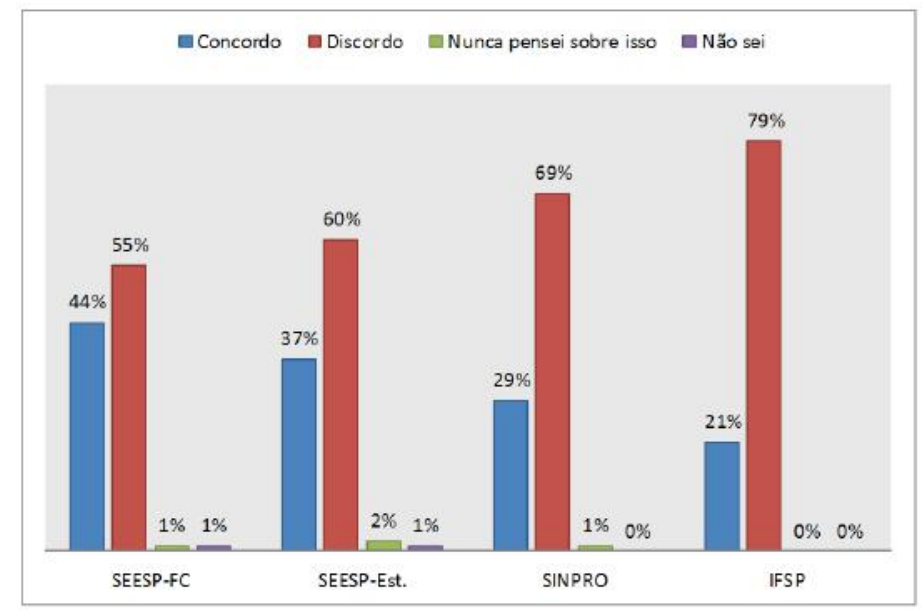

Fonte: Coelho, 2014. 
Para o outro enunciado da bateria apresentada na questão 27.1: "Se tiver a mínima possibilidade de um risco grave, não permitiria a aplicação da novidade científica ou tecnológica", a média de professores que afirmaram concordar parcialmente ou plenamente foi de $70,17 \%$. Discordam da proposição, $25,22 \% ; 3,35 \%$ nunca pensaram sobre isso e $1,5 \%$ não souberam responder.

Dentre os docentes, os do IFSP parecem ser os mais receosos em aceitar a novidade, já que aproximadamente $76 \%$ responderam que não permitiram a aplicação de novidade científica.

Os menos precavidos em relação às novidades foram os docentes vinculados à rede regular da SEESP, já que 30\% discordaram de rejeitar a inovação científica ou tecnológica frente à possibilidade de risco, conforme demonstrado no gráfico 6.

Gráfico 6: Percentual de rejeição de C\&T frente a possibilidade de risco

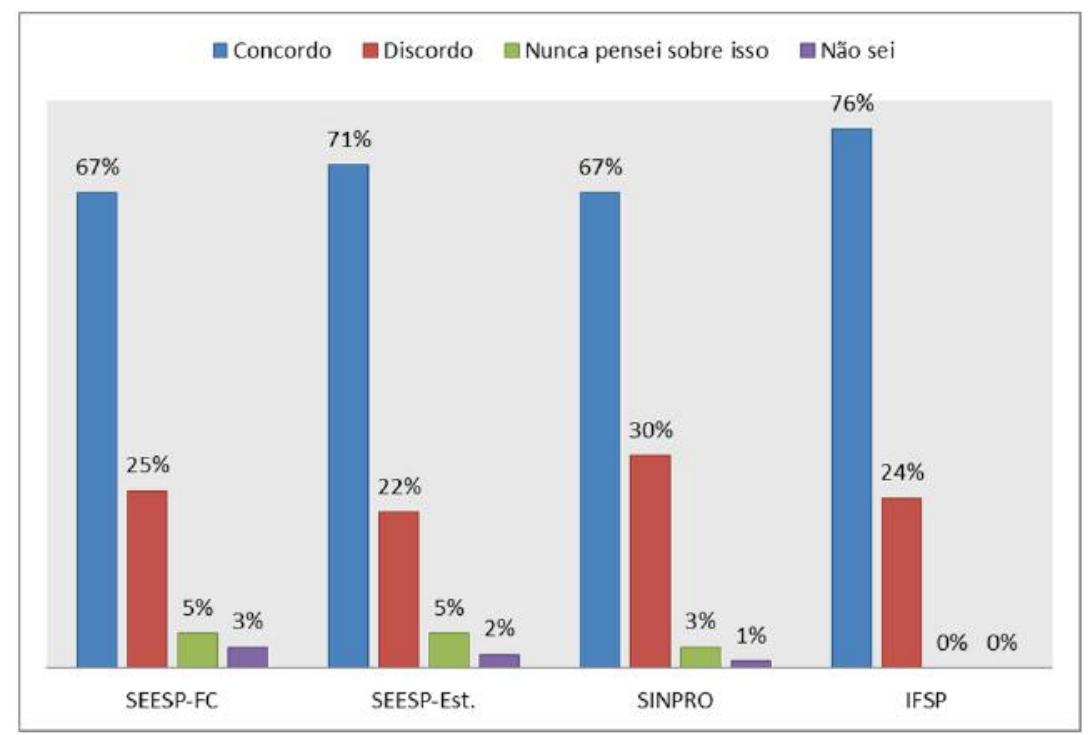

Fonte: Coelho, 2014.

A média dos docentes que afirmaram rejeitar inovação de C\&T frente a algum tipo de risco é compatível com a média da população geral. O resultado nesta questão, quando aplicada à população do estado de São Paulo, foi que 76,2\% também rejeitariam o benefício nessas condições.

Por outro lado, o percentual dos docentes que discordam da proposição, ou seja, que aceitariam as novidades ainda que houvesse risco, é de $25,2 \%, 17,2 \%$ maior do que os entrevistados da população em geral ( $8,0 \%)$. Dentre os que não se posicionaram, o percentual dos professores é $9,9 \%$ menor do que os do outro grupo de entrevistados $(14,7 \%)$.

\section{Conclusão}

Pesquisas de percepção pública da ciência têm avaliado os hábitos informacionais da população acerca de temas científicos e tecnológicos como forma de avaliar os meios utilizados, a frequência e o grau de informação sobre $\mathrm{C} \& \mathrm{~T}$ consumida. $\mathrm{O}$ desenvolvimento de um indicador a partir de tais perguntas, o Índice de Consumo de Informação Científica (Icic), por sua vez, permite o agrupamento dos entrevistados em categorias de acordo com tais parâmetros e, dessa forma, a análise de como o consumo de informação sobre C\&T varia entre diferentes públicos, bem como tais grupos se diferenciam ou assemelham-se entre si em relação a outras variáveis, como, por exemplo, suas atitudes e valoração de temas relacionados a C\&T. 
O estudo realizado com professores de ensino médio do estado de São Paulo permitiu confrontar tais resultados com os de outras pesquisas realizadas com diferentes públicos da mesma região. Percebe-se, dessa forma, que o grupo de docentes apresenta um nível de consumo de informação científica superior ao da população em geral, talvez como consequência das práticas relacionadas às suas atividades profissionais, da formação recebida, ou até mesmo pelo interesse pessoal dos indivíduos que escolheram a carreira docente.

Um nível mais elevado de consumo de informação sobre temas científicos e tecnológicos, no entanto, não significa menor senso crítico em relação a aplicações da C\&T. Os professores, apesar de apresentarem uma visão otimista da ciência, como observado também na população em geral e em jovens estudantes, demonstram cautela ao reconhecerem possíveis riscos oferecidos por implicações da ciência ou de novos desenvolvimentos tecnológicos.

Quando se trata da participação social em tomadas de decisão sobre temas relacionados a C\&T, o conhecimento especializado parece ter menos importância do que a garantia da ação democrática. Os professores, assim como os demais públicos analisados, consideram que os cidadãos devem ser ouvidos a respeito de implicações científicas e tecnológicas que afetem as suas vidas, e que tais decisões não podem ficar a cargo somente de especialistas. 


\section{REFERÊNCIAS}

Bazzo, W. (1998). A Ciência, Tecnologia e Sociedade: e o contexto da educação tecnológica. Florianópolis, Brasil: Ed. da UFSC.

Coelho, M. (2014). Percepção dos Professores de Ensino Médio sobre Temas relacionados a $C \& T$. Disponível em: $<$ https://ppctspro.wordpress.com/>.

CRISP. (2010). Chinese Public Understanding of Science and Attitudes towards Science and Technology. Beijing, China: CRISP. Disponível em:

$<$ http://www.cast.org.cn/n35081/n35473/n35518/12451858.html $>$.

CETIC (2011). TIC Educação-Professores. Disponível em: http://cetic.br/tics/educacao/2011/professores.

COLCIENCIAS. (2005). Primera encuesta sobre la imagen de la ciencia y la tecnología en la población colombiana, 1994. Disponível em:

$<$ http://www.upf.edu/pcstacademy/docs/EncuestaColombia.pdf $>$.

FAPESP. (2005). Indicadores de ciência, tecnologia e inovação em São Paulo, 2004. São Paulo, Brasil: FAPESP. Cap. 12.

- (2011) Indicadores de ciência, tecnologia e inovação em São Paulo, 2010. São Paulo, Brasil: FAPESP, Cap. 12.

FECYT. (2006). Percepción social de la ciencia y la tecnología en España-2004. Madrid, Espanha: Fecyt. Disponível em: $<$ http://www.oei.es/salactsi/percepcion.pdf $>$.

FECYT-OEI-RICYT. (2007). Cultura científica em Iberoamérica. Encuesta en grandes núcleos urbanos. Madrid, Espanha: Fecyt. Disponível em: <http://www.fvc.org.br/pdf/ estudocomputador-internet.pdf>.

Gouw, A. M. (2013). As opiniões, interesses e atitudes dos jovens brasileiros frente à ciência: uma avaliação de âmbito nacional. Disponível em:

$<$ www.teses.usp.br/teses/.../ANA_MARIA_SANTOS_GOUW_rev.pdf $>$.

Giddens, A. (1991). As Consequências da Modernidade. São Paulo, Brasil: Ed. Unesp.

MCTI. (2006). Pesquisa de Percepção Pública da Ciência. Disponível em:

$<$ http://www.cnpq.br/documents/10157/c52098dc-9364-4661-a8a9-d99c0b2bb9ef $>$.

NISTEP. (2013). Japanese Science and Technology Indicators 2012. Research Material 214. Disponível em: $<$ http://www.nistep.go.jp/en/?page id=52>.

NSF. (2008). Disponível em: $<$ http://www.nsf.gov/statistics/seind08/c7/c7h.htm $>$.

OEI. (2011). Los Estudiantes y la Ciencia: encuesta a jovenes ibero-americanos. Polino, C. (Org.). Buenos Aires, Argentina. Disponível em: $<\underline{\mathrm{http}} / /$ www.oei.es/salactsi/ libroestudiantes.pdf $>$.

SECYT. (2007). La percepción de los argentinos sobre la investigación científicas en el país. In: Segunda Encuesta Nacional De Percepción Pública de la Ciencia. Buenos Aires, Argentina: Secyt.

Vogt, C. e Polino, C. (Orgs.). (2003). Percepção pública da ciência, Resultados da Pesquisa na Argentina, Brasil, Espanha e Uruguai. São Paulo, Brasil: Editora Unicamp.

\section{SOBRE OS AUTORES}

Márcia Azevedo Coelho: Pesquisadora colaboradora do Laboratório de Estudos Avançado em Jornalismo da Universidade Estadual de Campinas (Labjor/Unicamp, Brasil) e pós-doutoranda em percepção pública da ciência (Bolsista Fapesp-Processo 2013/04008-6).

Ana Paula Morales: Pesquisadora colaboradora do Labjor/Unicamp, doutoranda em política científica e tecnológica pela Unicamp e assessora de comunicação da Universidade Virtual do Estado de São Paulo (Univesp), Brasil.

Carlos Vogt: Coordenador do Labjor/ Unicamp e presidente da Univesp, Brasil. 